Relations industrielles

Industrial Relations

\title{
The Practice of Collective Bargaining, par Beal et Wickersham, Edition revisée, 1963, Richard D. Irwin, Inc., Homewood, Illinois, 772 pages.
}

\section{Jean-Réal Cardin}

Volume 19, numéro 1, janvier 1964

URI : https://id.erudit.org/iderudit/1021384ar

DOI : https://doi.org/10.7202/1021384ar

Aller au sommaire du numéro

Éditeur(s)

Département des relations industrielles de l’Université Laval

ISSN

0034-379X (imprimé)

1703-8138 (numérique)

Découvrir la revue

Citer ce compte rendu

Cardin, J.-R. (1964). Compte rendu de [The Practice of Collective Bargaining, par Beal et Wickersham, Edition revisée, 1963, Richard D. Irwin, Inc., Homewood, Illinois, 772 pages.] Relations industrielles / Industrial Relations, 19(1), 132-133. https://doi.org/10.7202/1021384ar

Tous droits réservés (C Département des relations industrielles de l’Université Laval, 1964
Ce document est protégé par la loi sur le droit d'auteur. L'utilisation des services d'Érudit (y compris la reproduction) est assujettie à sa politique d'utilisation que vous pouvez consulter en ligne.

https://apropos.erudit.org/fr/usagers/politique-dutilisation/ 
pos considérés comme relevant de la même méthodologie; ces deux secteurs sont, d'une part, l'économie du travail, et d'autre part, les relations industrielles dans leurs dimensions institutionnelles.

Se trouvent ainsi liées l'étude de la détermination des salaires et de l'emploi, et celle du syndicalisme et de la négociation collective en tont qu'institutions sociales.

L'ouvrage de Gitlow fait de plus appel oux toutes dernières recherches dans le domaine de l'économie et de la sociologie industrielles, ce qui en fait un témoignage des plus à point sur le degré des connaissances acquises jusqu'ò maintenant en la matière.

La première et la dernière partie du volume constituent une espèce de bilan du monde du travail et des problèmes d'ordre généroux qui sont les siens au sein d'une société industrielle qui évolue sons cesse.

Les parties II, III, IV, V, et VI sont consocrées respectivement ou trade-unionisme en tant qu'institution sociale, ì la discussion des théories relatives à la détermination des salaires, à la convention collective institutionnalisée, ò la fonction de plein-emploi et aux lois protectrices du trovail.

Des ouvroges suggérés sont indiqués d la fin de chaque chapitre, ce qui constitue, tout au long du traité, une bibliographie sélective très utile pour l'étudiont.

En somme, nous avons affaire à une étude vraiment compréhensive et bien équilibrée de l'ensemble des problèmes de relotions industrielles tels qu'ils se posent au spécialiste contemporain. Bien qu'il ne s'agisse que d'un « textbook \d'envergure générale, et non pas d'un ouvrage de recherche poussée et spécialisée, le travail de Gitlow se situe ovec ovantage parmi les volumes de référence les mieux faits et les plus utiles aux étudiants en économie du travail et en relotions industrielles.

\section{Jean-Réal Cardin}

The Practice of Collective Bargaining, par Beal et Wickersham, Edition revisée, 1963 Richord D. Irwin, Inc., Homewood, Illinois, 772 poges.

Il s'agit encore ici, d'un ouvrage appartenant d̀ la série des textes en économie du travoil publiée par la maison d'édition Irwin.
Lo présente édition se distingue de lo première, celle de 1959, en ce qu'elle permet oux auteurs de serrer de plus près I'analyse des concepts théoriques fondant l'étude de la négociation collective en Amérique du Nord, et oussi en ce qu'elle ojoute quant à la discussion des récents développements survenus en ce domaine aux EtatsUnis.

Le processus de la négociation collective y est traité, comme le déclarent les auteurs dans la préfoce de l'ourrage, selon deux situations-types: a) celle de l'industrie ò base «artisanale \ et du syndicalisme «de métiers »; b) celle des < industries de masses $\gg$ et du syndicolisme de type cindustriel $\gg$.

Les situations particulières, ne se conformant pas entièrement aux deux \&modèles \$ industriels cités plus hout, n'en sont pas pour outant négligées. On y traite donc des formes qu'assume la négociation collective non seulement dans le secteur manufacturier, mais oussi dons les secteurs primaires et tertiaires de l'économie.

Un des aspects vraiment intéressants de ce monuel, est l'analyse que les outeurs font des effets récents et prévisibles sur la convention collective contemporaine, des changements assez spectaculaires qui sont omorcés depuis quelques années et qui continuent de se manifester de foçon dromotique, dans la structure des emplois aux Etats-Unis, dû à l'automation et à la tronsformation de l'entreprise.

Le triangle classique des relations du trovail est respecté par les auteurs, car la négociation et la convention collectives y sont étudiées dans le cadre des changements survenus depuis certain nombre d'années, à la fois dans l'industrie, les pouvoirs publics et le travail organisé.

En particulier, on $y$ insiste sur les implications nouvellés de la stratégie de la négociation, des toctiques patronales et syndicales en motière de grèves, de règlements des conflits, d'action politique, quant à la notion d'intérêt public et quant aux ottitudes gouvernementoles qui en dépendent.

Comme beoucoup d'autres manuels en la matière, mais peut-être d'une foçon plus intensive et plus ò point, le présent ouvrage utilise la méthode des bibliographies choisies en fonction des sujets traités, offre des «problèmes d da la recherche personnelle de l'étudiant, et lui présente des <cos $\$$ con- 
crets utilisables pour fins de discussion et d'onalyse.

Enfin, les principoux textes législotifs pertinents ou sujet sont fournis en oppendice, soit dans leur teneur totale, soit en dé larges extraits.

Somme toute un des manuels les mieux faits et parmi les plus utiles, outant aux proticiens qu'aux étudiants en lo matière.

\section{Jean-Réal Cordin}

Le Statut des Entreprises publiques, par A G. Delion, Collection de l'Administration nouvelle, Berger-Levrault, Paris, 1963, 268 pages.

L'on n'a plus à démontrer, il me semble, l'importance des entreprises publiques dans la plupart des pays y compris ceux de I'Occident. En ce qui a trait à la France, André DELION a déjà publié plusieurs études sur le sujet, notomment, PEtat et les entreprises publiques (Sirey, Poris, 1959).

Dans Le Statut des Entreprises publiques $\gg$, I'auteur examine systématiquement et fort judicieusement les principales interrelations entre d'une part, l'entreprise publique et d'autre part, les organismes de contrôle que sont le porlement, le gouvernement et le Conseil d'Etat. En guise d'introduction, DELION donne quelques notes historiques et indique les règles relatives à la création, d̀ la notionalisotion et d̀ la disparition des entreprises publiques. L'ouvroge est très à point et fort bien rédigé

L'opproche est foncièrement formelle et juridique. Ce qui explique sans doute pourquoi I'on a ignoré le rôle des partis et celui de l'opinion publique Les discussions et les critiques sont rares. Les préoccupations d'ordre administrotif, politique et économique sous-jocentes sont rarement exprimées. II existe à cet égard un contraste frappant entre l'ouvrage de DELION et certaines études des onnées d'après guerre. notamment celles de Ventanat, de Juliot de la Morandière et de Lavergne qui mettaient en cause, sous différents aspects, l'idée même de la nationalisation. Cette idée étont mointenant devenue occeptoble il appartient ou juriste de formuler les nouvelles règles fondamentales d'organisation générale, c'est-à-dire, le stotut.

II va sans dire que I'on peut difficilement comporer le statut des entreprises publiques en Fronce et au Québec. Même si l'on fait obstraction des différences d'ordre quantitatif qui sont considérobles (en 1963, lo France comptait 150 entreprises publiques, le gouvernement canadien une quarontaine et le Québec une dizaine) il n'en reste pos moins que le contexte global est loin d'être identique. Lo planificotion économique est ici embryonnaire, les responsabilités sont portagées entre les collectivités provincioles et centrales, enfin la gestion publique commerciole est encore une source de controverses.

Sous l'angle restreint des rapports entre les entreprises publiques et les autorités de tutelle, il est vrai que nous observons ici oussi le principe de l'autonomie de gestion. Par contre, les moyens de contrôle diffèrent sensiblement. II n'y a pos ou Québec, par exemple, de commissaires de gouvernement, ni Commissions de Vérification des comptes des entreprises publiques, ni bien entendu, de Conseil d'Etot. De plus, les conseils d'administration n'obéissent pos ò lo formule de composition tripartite. Quont d la surveillance parlementaire, la nôtre frise I'insignifiance. L'Assemblée Législative n'a élaboré oucun méconisme spécial de contrôle. Evidemment, l'on peut expliquer ces différences par une foule de raisons dont certaines sont attribuables oux structures même de l'administration générale. Par contre, il fout bien dire qu'd mon sens, I'on a peu ressenti ici la nécessité d'une surveillonce plus étroite tout simplement à cause du petit nombre d'entreprises publıques. Le besoin devrait éventuellement créer l'organe.

\section{André Gélinas}

\section{Economic Analysis and Industrial Manage-} ment, par Jacques Lesourne, Prentice Holl, Inc., Englewood Cliffs, N.J., 1963, 631 pages.

$\mathrm{Ce}$ volume a déjò paru en françois en 1958 sous le titre \& Technique Economique et Gestion Industrielle $>$ II constitue une lecture probablement des plus profitables pour le futur ingénieur-économiste. En effet, nous croyons que ce livre s'odresse surtout aux ingénieurs qui se dirigent vers l'odministration de l'entreprise et qui partont désirent ocquérir une connaissonce de la théorie économique appliquée d̀ la gestion industrielle. Beaucoup d'économistes peuvent égolement tirer profit de lo formulation des principes économiques en termes mothématiques et statistiques. II ne 